\title{
Endoscopically Observed Outer Membrane Color of Chronic Subdural Hematoma and Histopathological Staging: White as a Risk Factor for Recurrence
}

\author{
Masahito KATSUKI, ${ }^{1}$ Yukinari KAKIZAWA, ${ }^{1}$ Naomichi WADA, ${ }^{1}$ \\ Yasunaga YAMAMOTO, ${ }^{1}$ Toshiya UCHIYAMA, ${ }^{1}$ Toshitsugu NAKAMURA, ${ }^{2}$ \\ and Masahide WATANABE ${ }^{2}$ \\ ${ }^{1}$ Department of Neurosurgery, Suwa Red Cross Hospital, Suwa, Nagano, Japan; \\ ${ }^{2}$ Department of Pathology, Suwa Red Cross Hospital, Suwa, Nagano, Japan
}

\begin{abstract}
Endoscopic treatment for chronic subdural hematoma (CSDH) has been reported, but endoscopic findings of CSDH have not been thoroughly investigated. This study aimed to elucidate the relationship between endoscopic findings and CSDH recurrence. Furthermore, it examined the association between Nagahori's histopathological staging of CSDH and outer membrane color. Here, we retrospectively analyzed the operative videos of 70 patients with CSDH. The endoscopic findings were investigated, and their correlations with CSDH recurrence, the reduction ratio of the midline shift, and hematoma thickness on day 30 after the operation were analyzed. The outer membrane was white in 21 cases, yellow in 25 cases, and red in 24 cases. CSDH recurred in three $(4.2 \%)$ patients, all of whom had a white outer membrane (adjusted odds ratio, $18 ; 95 \%$ confidence interval, $1.6-20.6 ; P=0.007$ ). The other endoscopic findings were not significantly related to CSDH recurrence, extent of the reduction ratio of the midline shift, or hematoma thickness. The outer membrane colors of white, red, yellow, and white almost corresponded to the histopathological staging from type I to IV in order. Our findings suggest that a white outer membrane is a risk factor for recurrence; these colors may represent the extent of inflammation related to the evolution of CSDH estimated from the histopathological findings.
\end{abstract}

Key words: chronic subdural hematoma, neuroendoscope, recurrence, outer membrane, histopathology

\section{Introduction}

Although chronic subdural hematoma (CSDH) is one of the most common diseases seen by neurosurgeons, its pathogenesis remains unclear. CSDH is usually associated with good recovery with burr hole irrigation and postoperative drainage under local anesthesia without using endoscopes. ${ }^{1-3)}$ This simple procedure elicits a satisfactory therapeutic effect, so few reports have discussed the endoscopic findings of $\mathrm{CSDH} .{ }^{4,5)}$

This study aimed to determine the importance of such endoscopic findings for reducing the recurrence of CSDH. Among several endoscopic findings, we emphasize the importance of the outer membrane by focusing on its color, which can be observed only

Received September 5, 2019; Accepted November 5, 2019

Copyright $@ 2020$ by The Japan Neurosurgical Society This work is licensed under a Creative Commons AttributionNonCommercial-NoDerivatives International License. endoscopically. Nagahori et al. ${ }^{6)}$ previously reported the histopathological staging of $\mathrm{CSDH}$, classified according to inflammation extent. Accordingly, here we determined the correlation between outer membrane color and histopathological staging and discussed the pathogenesis of CSDH and its recurrence.

\section{Materials and Methods}

We retrospectively retrieved data for all 119 patients with CSDH who were admitted to our hospital between February 2016 and July 2019. Each provided written informed consent for their inclusion in this study, which was approved by the hospital's research ethics committee. The diagnosis of CSDH was based on clinical history and the presence of subdural hematoma as determined on computed tomography (CT) images. Surgical intervention was performed when hematoma thickness was sufficient to compress the brain and when the neurological symptoms were obvious and due to the CSDH. In 
patients with bilateral hematomas, the operation was performed on both sides. However, when the hematoma on one side was much larger than that on the other side and the neurological symptoms seemed to be due to the more massive hematoma, we performed burr hole surgery unilaterally on the responsible side.

We performed single burr hole surgery with irrigation using artificial cerebrospinal fluid and postoperative drainage under local anesthesia with sedation for all 119 patients regardless of the endoscopic procedure or not. We made a burr hole on the part of the cranium where the hematoma was the thickest. The outer membranes were removed and sent for histopathological analysis whenever possible. The specimens were formalin-fixed and stained with hematoxylin and eosin. After irrigating the hematoma cavity, we used a rigid endoscope (Hopkins II, $4.0 \mathrm{~mm}, 30^{\circ}$ angle; Karl Storz, Tuttlingen, Germany) to observe the entire circumference of the hematoma cavity, paying attention to the brain surface, the dura, and the border between the inner and outer membranes after correcting for its white balance. We endoscopically destroyed the septum and the membranous structures of the septated CSDH using a suction tube and scissors under endoscopic observation so the CSDH was not divided into compartments by the septum and efflux of the hematoma could be achieved (Fig. 1G). When apparent bleeding in the hematoma cavity was observed, the bleeding was endoscopically coagulated using flexible bipolar electrocautery. The soft hematoma was suctioned by a suction tube (Figs. 1A and 1B). These endoscopic procedures were performed when they seemed to contribute to hematoma removal and could be performed safely. The drainage tube was removed within 2 days after the operation. All of the patients took the Japanese herbal kampo medicine goreisan postoperatively, which acts as a hydragogue by inhibiting aquaporin expression and could prevent CSDH recurrence. ${ }^{7}$

We performed 119 operations for the first CSDH and another seven for recurrence and two for re-recurrent CSDH. Of the 119 patients, one patient died of pneumonia, while two others were moved to other clinical facilities due to their main diseases, preventing follow-up of CSDH recurrence. Therefore, the operations of 116 patients were investigated. However, we retrieved the operative videos of 70 of the 116 patients. Of those 70 patients, the three
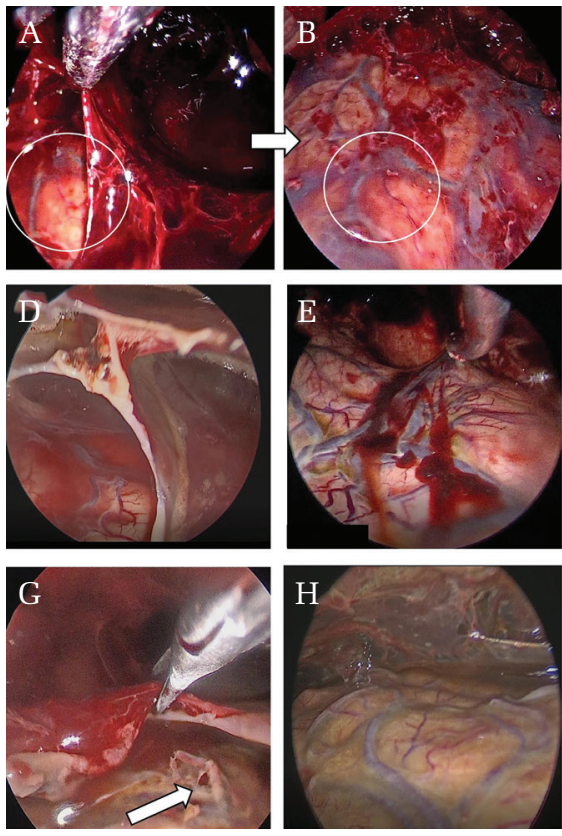
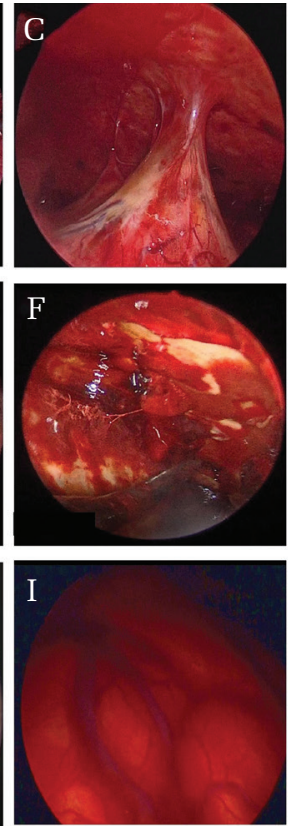
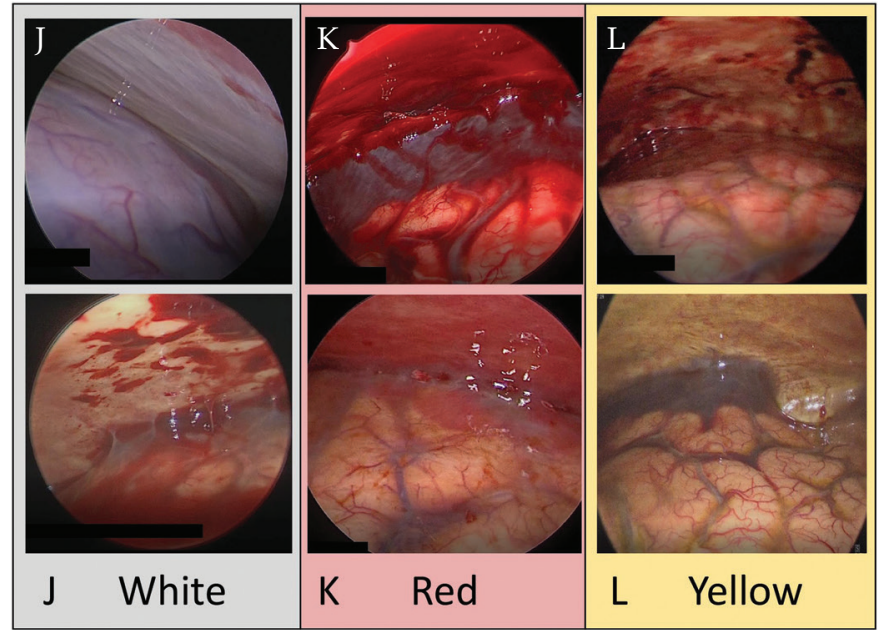

Fig. 1 Endoscopic findings in the chronic subdural hematoma cavity. A soft hematoma on the inner membrane was carefully suctioned by a suction tube to observe the hematoma cavity in detail (A and B). The circles in A and $B$ show the same parts. Trabecula (C), septum (D), clots adhering to the inner membrane (E), clots adhering to the outer membrane (F), an apparent tear of the arachnoid mater (G), a yellow inner membrane (H), and a red membrane (I). The septum was endoscopically divided using scissors (G). The color groups of the outer membrane (J-L). The white group presents an evenly white outer membrane without capillaries and with/without patchy clots like erythema and purpura (J). The red group presents with an evenly red or pink outer membrane with/ without hemorrhage or hematoma adhering to the outer membrane (K). The yellow group presents as yellow or orange but not evenly red or white outer membrane with capillaries developing apparently (L). 
patients underwent reoperation due to recurrence; thus, their first operative videos were investigated. We could not retrieve the operative videos of the other 46 patients because we performed CSDH surgery as an emergency operation, so the endoscope could not always be used depending on the state of the operating room and its staff. Therefore, 70 patients were included in the final analysis, and outer membranes from 22 of them were collected as histopathological specimens.

We collected data regarding the patients' physiological symptoms and medical history: age, sex, chief complaint, site of hematoma, period between latest trauma and operation, and whether they were taking oral antithrombotic drugs.

Hematoma density was evaluated on CT images before the surgical intervention according to the classification system developed by Lanksch et al. ${ }^{8)}$ Hematoma thickness and midline shift were measured in the pre- and post-operative periods (on day 30). Hematoma thickness was measured on the slice with the maximum value. The midline shift, regardless of unilateral or bilateral hematoma, was determined by measuring the distance from the midline on the axial CT scan to the midline structures, such as the falx or the third ventricle. In patients with bilateral hematoma, the left and right thicknesses were measured and the average value was used in the statistical analysis. The reduction ratios of the midline shift and hematoma thickness on day 30 after the operation were calculated using the following formula:

$$
\left\{\begin{array}{l}
1-\frac{\begin{array}{l}
\text { Postoperative midline shift } \\
\text { (hematoma thickness) }
\end{array}}{\text { Preoperative midline shift }} \\
\text { (hematoma thickness) }
\end{array}\right\}
$$

We examined the operative videos in detail and retrieved the endoscopic findings of the hematoma cavities after providing sufficient irrigation. Five neurosurgeons reviewed the videos. The questioner and investigators were blinded to the outcome. From the endoscopic videos, we determined the presence of trabecula, septum, clot adhering to the inner/outer membrane, and apparent tear of the arachnoid mater. Trabecula was defined as a thin structure projecting from the outer membrane to the inner membrane ${ }^{5)}$ or membranous structure surrounding the arachnoid adhering to the dura. The septum was defined as a membranous structure dividing the cavity into small compartments. We also determined the color of the inner and outer membranes. The color of the inner membrane was divided into yellow or red, representing yellow and red brain surface covered by the inner membrane (Figs. 1C-1I). The color of the outer membrane was divided into white, yellow, or red. The white group showed an evenly white outer membrane without capillaries and with/without patchy clots like erythema and purpura. The yellow group showed yellow or orange with apparent capillary development but not an evenly red or white outer membrane. The red group showed an evenly red or pink outer membrane with/without hemorrhage or hematoma adhering to the outer membrane. We focused on the overall color of the outer membrane itself as the background after a thorough irrigation. Clots, hemorrhage, and hematoma were not considered in the color determination. A mixed-color type (ex: some areas were white, other areas were red) was classified to the dominant color of the whole outer membrane (Figs. 1J-1L). The presence of each finding and color were decided by the majority of the answers of the five investigators.

Two neurosurgeons classified the histological features into four types as described by Nagahori ${ }^{6)}$ focusing on membrane thickness, capillary quantity, intra-membranous hemorrhage, infiltrated cells, and connective tissue: type I (non-inflammatory membrane) containing immature fibroblasts and collagen fibers associated with very slight or sparse cell infiltration and neocapillaries; type II (inflammatory membrane) showing a single-layer membrane with high cellularity and vascularization; type III (inflammatoryhemorrhagic membrane) consisting of a multi-layered membrane with high cellularity, large neocapillaries, and scattered infiltrated cells; and type IV (scarinflammatory membrane) consisting of connective tissue composed of mature fibroblasts and collagen but scarce neocapillaries. It was hypothesized that the outer membrane of CSDH evolves from type I to IV in order, with reference to CSDH pathophysiology. ${ }^{6}$ )

We performed a statistical analysis of the associations of these factors with the reduction ratio of midline shift and hematoma thickness on day 30. The relationships between these factors and recurrence requiring reoperation and the relevance between the outer membrane color and histopathological staging were also investigated.

We used the Mann-Whitney $U$ test to examine non-parametric data of the two groups, Fisher's exact test for categorical variables of the two groups, Spearman's coefficient test for non-parametric variables of the two groups, and the Kruskal-Wallis test for non-parametric data of more than two groups. The inter-rater reliabilities of the outer membrane color and histopathological measurements were tested by Fleiss's kappa test. In all analyses, values 
of $P<0.05$ were considered statistically significant. We conducted these analyses using SPSS software version 24.0.0 (IBM Corp., Armonk, NY, USA).

\section{Results}

\section{Clinical characteristics, CT findings, and endoscopic findings}

The total 116 patients with/without endoscopic procedures consisted of 35 women and 81 men, a mean age of 78 (range, 29-95) years. The neurological symptoms on admission were improved after surgery in all patients. Seven patients $(6.0 \%)$ after the first surgery experienced recurrence and underwent repeat surgical intervention. The difference between the recurrence rate with endoscopy (three of 70 patients) and that without endoscopy (four of 46 patients) was not significant $(P=0.47)$.

Among the 70 patients with endoscopic operative videos, 52 were male. The mean age was 76.2 (range, 29-95) years. The hematoma was on the left in 20 and the right in 25; 25 had bilateral hematomas. Of them, five underwent surgical intervention for the left hematoma, four for the right hematoma, and 16 patients for both. Of the 43 patients for whom information was available, the mean period between the latest trauma and the operation was 51.5 (range, 3-180) days. Of the 70 patients for whom endoscopic operative videos were available, 22 had taken oral antithrombotic drugs. The mean preoperative midline shift was 7.6 (range, 0-18.6) $\mathrm{mm}$. The mean hematoma thickness was 23.7 (range, 8.4-40) mm (Table 1).

\section{Associations between variables and recurrence}

Three $(4.3 \%)$ of the 70 patients with endoscopic operative videos experienced recurrence and underwent reoperation. A white outer membrane was associated with a risk of CSDH recurrence [adjusted odds ratio, 18; 95\% confidence interval (CI), 1.6-206; $P=0.007$ ]. The other variables, including previously reported risk factors, showed no statistically significant relationship (Table 1). We also investigated the second endoscopic videos of the patients who underwent reoperation for the CSDH recurrence. The outer membranes in the second operation included two reds and one yellow of the three patients without re-recurrence.

\section{Associations between outer membrane color and histopathological staging}

Table 2 shows the association between outer membrane color and histopathological staging (fixed-marginal kappa for outer membrane color, 0.68; 95\% CI, 0.31-1.00; fixed-marginal kappa for histopathological staging, 0.85; 95\% CI, 0.53-1.00). The outer membrane colors of white, red, yellow, and white almost corresponded to the histopathological staging of type I-IV in order. White was the only color of type I, while red seemed specific to type III. Figure 2 shows the histopathological specimens and endoscopic findings of the same patients in order of histopathological staging.

Associations between outer membrane color, the density of hematoma, and presence of niveau

Table 2 also shows the association between outer membrane color, hematoma density, and the presence of niveau. High-density hematoma seemed related to white and red membranes, while iso-density hematoma seemed related to yellow membranes. Niveau was often seen with yellow membranes.

\section{Associations of variables with reduction ratios of hematoma thickness and midline shift}

The reduction ratio of the midline shift in patients taking antithrombotic drugs was significantly poorer than those not taking them $(P=0.02)$. Other variables, including previously reported risk factors, did not show significant relationships with the reduction ratio of midline shift or hematoma thickness (Table 3).

\section{Discussion}

\section{Endoscopic treatment for CSDH}

Endoscopic treatment for CSDH has been reported since the $1990 \mathrm{~s}^{9)}$ and is reported to be effective for some types such as septated $\mathrm{CSDH}^{10)}$ and organized CSDH. ${ }^{11)}$ Concerning the reduction of recurrence rate of CSDH, the use of an neuroendoscope enables safe removal of the clots, membranous structures, and trabecula in the hematoma cavity with direct visualization. ${ }^{4,5,12,13)}$ It also helps with confirming the washing effect of irrigation in the hematoma cavity, ${ }^{13)}$ inserting the drainage tube toward the frontal convexity to remove subdural air ${ }^{14)}$ avoiding cortical laceration due to blind manipulation, ${ }^{12)}$ coagulating the source of bleeding, ${ }^{15)}$ and tearing the inner membrane safely to promote brain expansion. ${ }^{16)}$ On the other hand, the disadvantages of endoscopic procedures include a potential to damage the cortical surface due to a blind angle or surgical error and extended operation time compared with procedures without a neuroendoscope. ${ }^{12,15)}$

Previous studies reported clots and trabecular structures as endoscopically observable risk factors for recurrence. ${ }^{4,5,10,17)}$ Clots would indicate recent bleeding and trabecular structures would be fragile, leading to bleeding. Of course, these factors are important for consideration as risk factors, but clot, 
Table 1 Clinical findings of the recurrence and non-recurrence groups

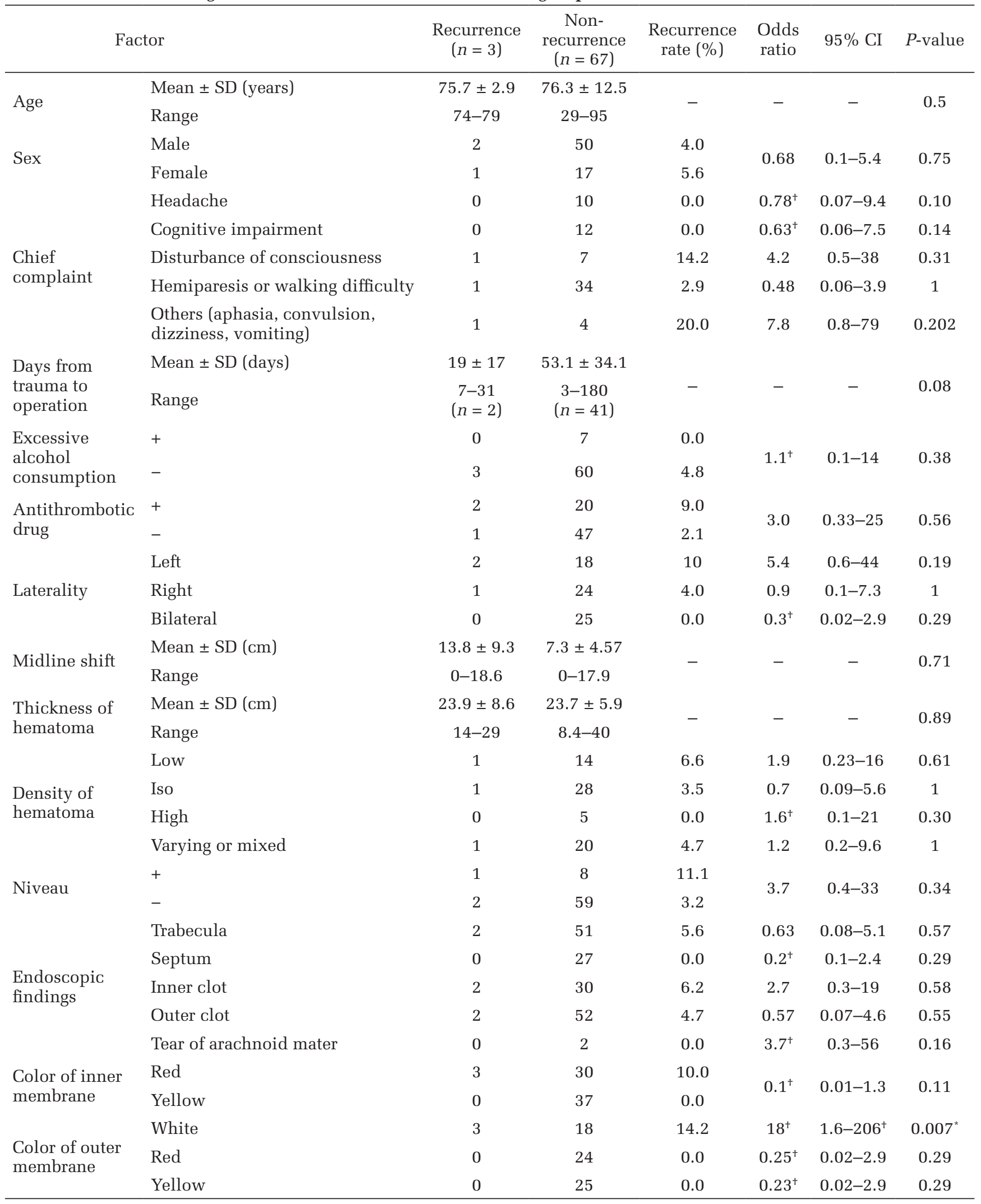

${ }^{*} \mathrm{P}<0.05 .{ }^{+}$Adjusted odds ratio. CI: confidence interval, SD: standard deviation. 
Table 2 Relationship between outer membrane color and histopathological staging, density of hematoma, and presence of niveau

\begin{tabular}{|c|c|c|c|c|c|c|c|c|c|}
\hline Number of cases & \multicolumn{4}{|c|}{ Histopathological staging } & \multicolumn{5}{|c|}{ Density of hematoma and presence of niveau } \\
\hline Color & Type I & Type II & Type III & Type IV & Low & Iso & High & Varying or mixed & Niveau (+) \\
\hline -White & 1 & 1 & 1 & 3 & 3 & 9 & 2 & 7 & 2 \\
\hline -Red & & 1 & 8 & & 7 & 8 & 3 & 6 & 2 \\
\hline -Yellow & & 1 & 2 & 4 & 5 & 12 & 0 & 8 & 5 \\
\hline
\end{tabular}

\section{Histopathological staging}

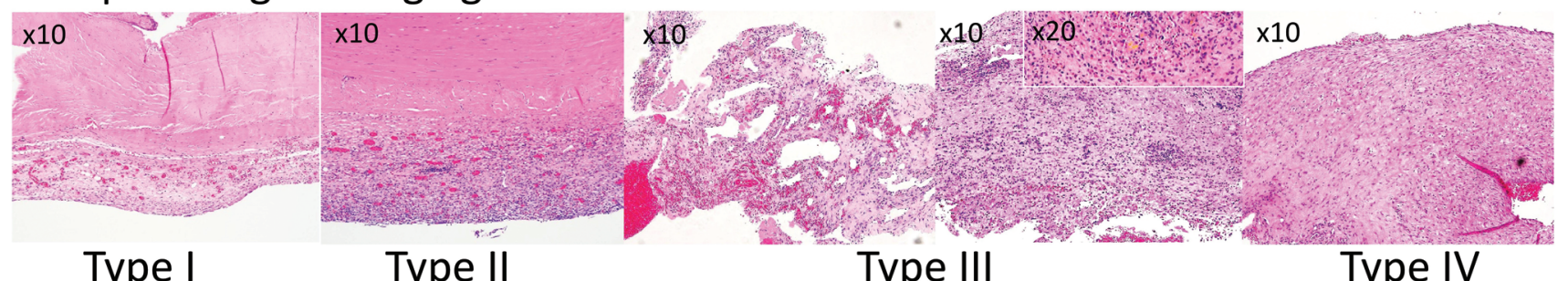

\section{Outer membrane color}

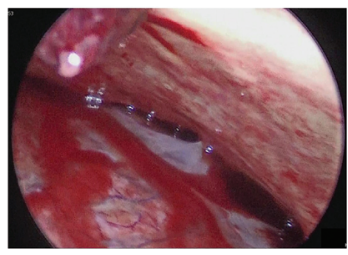

White

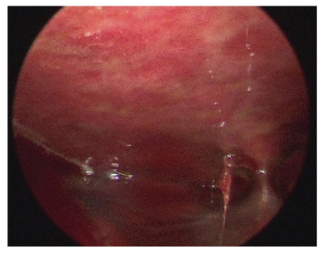

Red

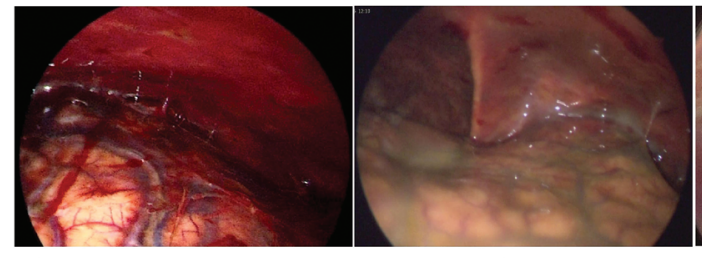

Yellow

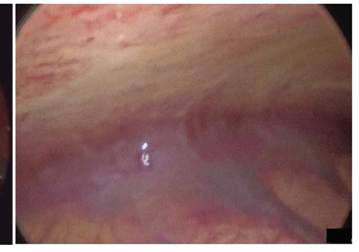

White

Fig. 2 The histopathological specimens and endoscopic findings of the same patients were lined up in order of histopathological staging. As the histopathological staging changes from type I to IV (upper row), the outer membrane color changes from white to red to yellow to white in order (lower row).

Table 3 Relationship of reduction ratio of midline shift and hematoma thickness with various factors

\begin{tabular}{|c|c|c|c|c|c|c|}
\hline & \multirow[t]{2}{*}{ Factor } & \multirow[b]{2}{*}{ No. } & \multicolumn{2}{|c|}{$\begin{array}{l}\text { Reduction ratio of midline shift } \\
\text { (1 month) }\end{array}$} & \multicolumn{2}{|c|}{$\begin{array}{l}\text { Reduction ratio of hematoma } \\
\text { thickness ( } 1 \text { month) }\end{array}$} \\
\hline & & & Mean \pm SD (\%) & $P$-value & Mean \pm SD (\%) & $P$-value \\
\hline Age & & & $(r=0.20)$ & 0.23 & $(r=-0.09)$ & 0.45 \\
\hline $\begin{array}{l}\text { Days from trauma to } \\
\text { admission }\end{array}$ & & & $(r=0.046)$ & 0.01 & $(r=-0.18)$ & 0.24 \\
\hline \multirow{2}{*}{ Antithrombotic drug } & + & 22 & $71 \pm 44$ & \multirow{2}{*}{$0.02^{*}$} & $54 \pm 16$ & \multirow{2}{*}{0.16} \\
\hline & - & 48 & $88 \pm 17$ & & $62 \pm 23$ & \\
\hline \multirow[t]{2}{*}{ Laterality } & Unilateral & 45 & $85 \pm 16$ & \multirow{2}{*}{0.54} & $59 \pm 22$ & \multirow{2}{*}{0.87} \\
\hline & Bilateral & 25 & $77 \pm 48$ & & $60 \pm 22$ & \\
\hline \multirow{2}{*}{ Density of hematoma } & & & & 0.86 & & \multirow{2}{*}{$\begin{array}{c}0.75 \\
(\mathrm{~K}-\mathrm{W} \text { test })\end{array}$} \\
\hline & & & & (K-W test) & & \\
\hline \multirow[t]{2}{*}{-Low } & + & 15 & $73 \pm 59$ & \multirow{2}{*}{0.64} & $56 \pm 27$ & \multirow{2}{*}{0.48} \\
\hline & - & 55 & $85 \pm 17$ & & $60 \pm 21$ & \\
\hline
\end{tabular}


Table 3 Relationship of reduction ratio of midline shift and hematoma thickness with various factors-Continued

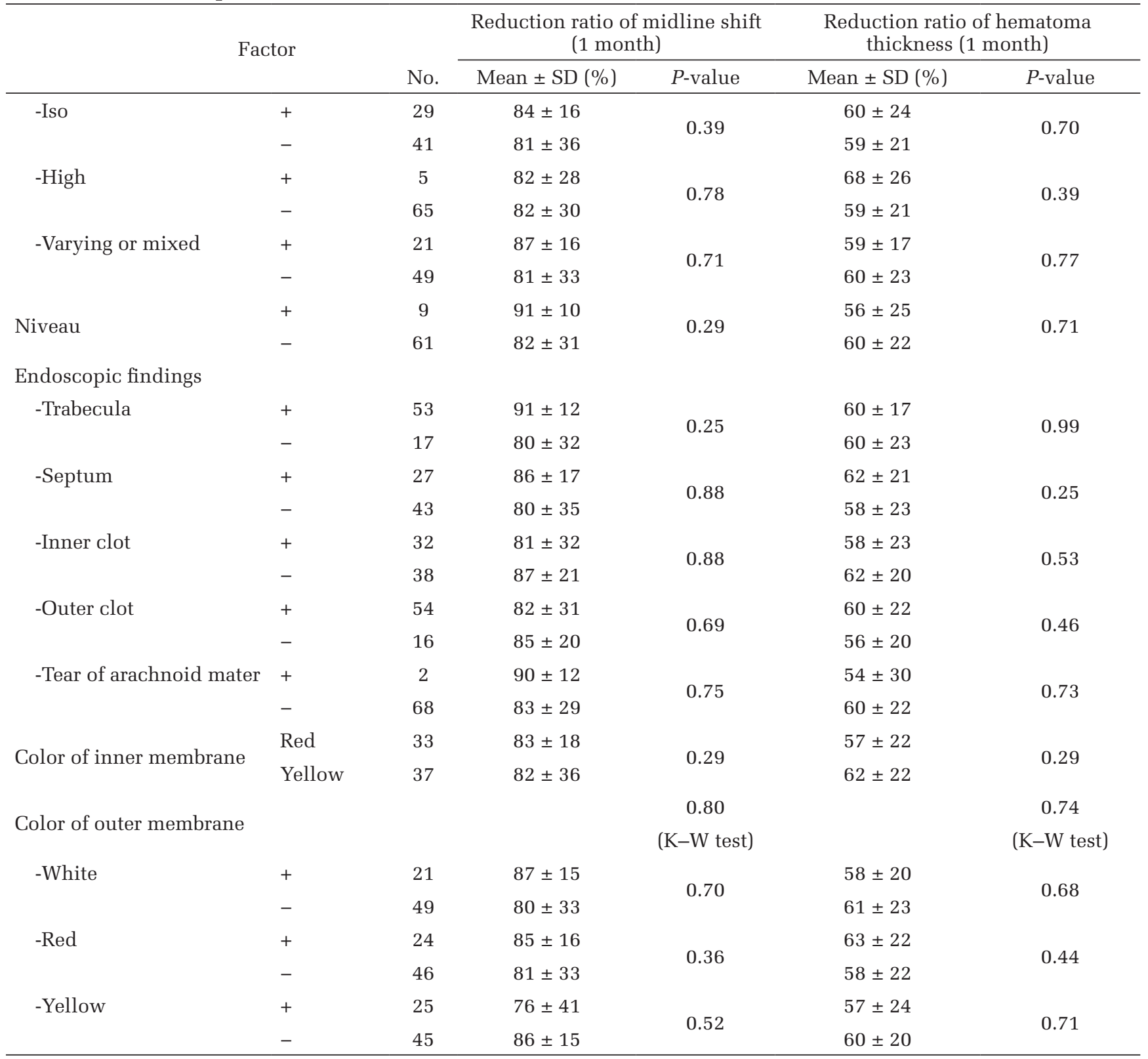

${ }^{*} \mathrm{P}<0.05$. K-W test: Kruskal-Wallis test, $r$ : correlation coefficient, SD: standard deviation.

trabecula, and the other findings are only parts of the whole hematoma structures. We gave weight to the color of the outer membrane as background and focused on the hematoma cavity in perspective. To the best of our knowledge, this is the first report on the association of outer membrane color with CSDH recurrence and histopathological staging.

\section{Pathophysiology of CSDH}

In 1946, Inglis ${ }^{18)}$ showed that the dura is lined with a layer of specialized modified connective tissue cells, which he named dural border cells. $\mathrm{CSDH}$ is considered to be induced by trauma in dural border cells. ${ }^{19)}$ The sustained inflammatory response around dural border cells in CSDH results in new membrane growth and fluid accumulation over time. ${ }^{17,20)}$ This is one of the most convincing hypotheses regarding the pathogenesis of CSDH. ${ }^{21,22)}$

Furthermore, the inner membrane consists of collagen and fibroblasts only and is considered nonfunctional in the development of CSDH. ${ }^{20)}$ On the other hand, the outer membrane, which contains layers of fibroblasts and collagen fibers with inflammatory cells such as neutrophils, lymphocytes, macrophages, and eosinophils ${ }^{17}$ is considered important for driving CSDH growth. 


\section{Histopathology of CSDH outer membrane}

Gandhoke et al. ${ }^{23)}$ reported that patients presenting with disturbance of consciousness tended to have type II membranes and hypothesized that the type II membrane represents an early stage of inflammation that results in a high rate of membranous exudation into the hematoma cavity. Park et al. ${ }^{24)}$ reported that hematoma thickness and midline shift were greatest in type II and reported large numbers of type II and IV cases with neurological deficits and recurrence. Therefore, type II membrane indicating inflammation seems crucial for CSDH development and recurrence.

\section{Outer membrane color}

Wakuta et al. ${ }^{4)}$ reported hematoma clots and spotty bleeding as endoscopically observable risk factors for recurrence. The outer membrane with hemorrhage and hematoma seemed red at a glance. However, we defined that the outer membrane color depended on the overall color of the outer membrane itself after irrigation and distinguished between the color and the presence of clots, hemorrhage, and hematoma.

Based on the results above, we assumed that the outer membrane color changes to white, red, yellow, and white in order as the histopathological staging changes from type I to IV. Type I membranes are thin, so it may be possible to see the white color of the underlying dura through the membrane. Type II membranes are single-layer membranes with high cellularity and numerous neocapillaries, while type III membranes are multi-layer membranes with large neocapillaries and hemorrhages. Therefore, type II and III membranes show reddish membranes with patchy clots, bleeding, and neocapillaries. Notably, type III membranes may be opaque red, obscuring the underlying white color of the dura. As the membranes change from type III to IV, the inflammation decreases. The capillaries degenerate and hemosiderin deposits, so outer membranes become yellow. Type IV membranes consist of nonfragile connective tissue and few neocapillaries; thus, they may appear as a thick white membrane. Our results also support this hypothesis (Fig. 2). However, the outer membrane color did not absolutely correspond to Nagahori's histopathological staging; rather, it seemed to change gradually with some overlap between histopathological stages.

We found that CSDH with a white outer membrane was more likely to recur compared with red and yellow membranes. In other words, a red outer membrane did not tend to recur, although it seemed to reflect high inflammation. We assumed that cases of white outer membranes suggested immature membranes (type I and pre-II) or fibrous membranes (type IV). Inflammation is in the process, and the membrane is evolving from type I to II, leading to $\mathrm{CSDH}$ development and recurrence. Otherwise, a white membrane may be a mature membrane such as type IV. The hematoma has compressed the brain for a long time, resulting in insufficient brain expansion and CSDH recurrence. ${ }^{16)}$

In addition to the color grouping associated with the histopathological findings, cerebrospinal fluid (CSF) communication between the hematoma cavity and the subarachnoid space may be another mechanism for white outer membrane organization. The washout effect of CSF may be responsible for rapid resolution of acute subdural hematoma. ${ }^{25)}$ Similarly, CSDH may also be washed out by CSF, and the outer membrane may look white despite continuous hemorrhaging. On the other hand, CSF is highlighted as a potential driver of CSDH formation. CSF accumulation evolves into subdural hygroma due to arachnoid tearing at the time of trauma or surgery, and it can potentially continue to leak into the subdural space ${ }^{17)}$ through a flap valve mechanism. ${ }^{26)}$ Delayed resorption of the subdural hygroma may elongate the bridging veins, resulting in hemorrhage into the subdural space ${ }^{27}$ and CSDH. Besides, CSDH occurs at a rate of $4-58 \%$ following traumatic subdural hygroma. ${ }^{26)}$ According to this theory, the dural border cell is not necessarily related to CSDH development. This is another hypothesis for why CSDH tends to recur in cases with a white outer membrane. In our study population, one patient experienced recurrence after unruptured aneurysm clipping surgery. CSF communication occurred between the subdural space and the subarachnoid space after dissection of the Sylvian fissure.

\section{Clinical application}

Our findings that CSDH with a white outer membrane tends to recur, and our classification of outer membrane color may contribute to clarification of the pathophysiology of CSDH in connection with histopathological classification. Clinically, a white outer membrane observed intraoperatively using a neuroendoscope can be an indicator that the patient may require careful follow-up. Oral anti-inflammatory drugs such as corticosteroids, ${ }^{28)}$ saireito (Japanese traditional medicine), ${ }^{29)}$ and antiangiogenic drugs such as angiotensin-converting enzyme inhibitors ${ }^{28)}$ can be considered.

\section{Limitation}

Our study population was small. To confirm the previously reported risk factors for recurrence, the minimum required number of samples should be calculated. We would have needed 160-1000 samples 
to reproduce the results suggesting trabecula, clot, ${ }^{4)}$ a highly dense hematoma on CT image, ${ }^{30}$ and bilateral hematoma ${ }^{31)}$ as previously reported risk factors. Also, we did not find a strong association between outer membrane colors and histopathological findings due to the small sample size. However, there are few reports on the endoscopic findings of CSDH and histopathological staging. Our study was preliminary, but we believe that it reports a new viewpoint.

Our data did not reveal the association between outer membrane color and the period from the latest trauma suggestive of CSDH progression. However, the period is unreliable because patients with CSDH tend to suffer repeated falls and have cognitive decline, so it is difficult to accurately determine the number of days from the latest trauma.

We did not observe only very white, red, and yellow membranes, we also saw pink, orange, and other intermediate colors. The change in histopathological staging over time is consecutive, so it is difficult to precisely categorize color. To maintain objectivity, we tried to use a color analysis software, but the result was too complicated to be interpreted. Despite correcting the white balance, it was very difficult to standardize the video image because of halation and variations in distance from the endoscope to the outer membrane. Therefore, this problem was solved by the majority vote of the five experienced neurosurgeons. The possibility that outer membrane color is relevant to only endoscopically treated cases and may not have the same implications for traditional multi-burr hole or mini-craniotomy strategies without endoscope use is another limitation. We believe that CSDH does not necessarily require endoscopic treatment.

\section{Conclusion}

We endoscopically observed the CSDH cavity and examined the associations between CSDH recurrence and endoscopic findings. A white outer membrane is a risk factor for CSDH recurrence. We concluded that the outer membrane color changes from white to red to yellow to white in order and that these colors may represent inflammation related to CSDH evolution estimated by histopathological findings.

\section{Contributions}

Yukinari Kakizawa, Naomichi Wada, Yasunaga Yamamoto for conception and design, all authors for drafting the article, Toshitsugu Nakamura, Masahide Watanabe for pathological analysis, Yukinari Kakizawa for supervision.

\section{Acknowledgments}

We would like to thank our medical staff for data acquisition.

\section{Conflicts of Interest Disclosure}

The authors report no conflicts of interest concerning the materials or methods used in this study or the findings presented in this paper. No sources of financial or material supported were received. The contents of this article were not published or presented previously.

\section{References}

1) Almenawer SA, Farrokhyar F, Hong C, et al.: Chronic subdural hematoma management: a systematic review and meta-analysis of 34829 patients. Ann Surg 259: 449-457, 2014

2) Liu W, Bakker NA, Groen RJ: Chronic subdural hematoma: a systematic review and meta-analysis of surgical procedures. J Neurosurg 121: 665-673, 2014

3) Ivamoto HS, Lemos HP, Atallah AN: Surgical treatments for chronic subdural hematomas: a comprehensive systematic review. World Neurosurg 86: 399-418, 2016

4) Wakuta N, Abe H, Nonaka M, et al.: Analysis of endoscopic findings in the chronic subdural hematoma cavity: bleeding factors in chronic subdural hematoma natural history and as predictors of recurrence. World Neurosurg 124: e241-e251, 2019

5) Shiomi N, Hashimoto N, Takeuchi H, Yamanaka T, Nakagawa N, Mineura K: [Endoscopic findings in chronic subdural hematoma]. No Shinkei Geka 30: 717-722, 2002 (Japanese)

6) Nagahori T, Nishijima M, Takaku A: [Histological study of the outer membrane of chronic subdural hematoma: possible mechanism for expansion of hematoma cavity]. No Shinkei Geka 21: 697-701, 1993 (Japanese)

7) Katayama K, Matsuda N, Kakuta K, et al.: The effect of Goreisan on the prevention of chronic subdural hematoma recurrence: multi-center randomized controlled study. J Neurotrauma 35: 1537-1542, 2018

8) Lanksch W, Kazner E, Grumme T (eds): Computed tomography in head injuries. Berlin Heidelberg, Springer, 1979

9) Hellwig D, Bauer BL: Minimally invasive neurosurgery by means of ultrathin endoscopes. Acta Neurochir Suppl (Wien) 54: 63-68, 1992

10) Berhouma M, Jacquesson T, Jouanneau E: The minimally invasive endoscopic management of septated chronic subdural hematomas: surgical technique. Acta Neurochir (Wien) 156: 2359-2362, 2014

11) Rodziewicz GS, Chuang WC: Endoscopic removal of organized chronic subdural hematoma. Surg Neurol 43: 569-572; discussion 572-573, 1995 
12) Mobbs R, Khong P: Endoscopic-assisted evacuation of subdural collections. J Clin Neurosci 16: 701-704, 2009

13) Shiomi N, Shigemori M: [The use of endoscopic surgery for chronic subdural hematoma]. No Shinkei Geka 33: 785-788, 2005 (Japanese)

14) Nakaguchi H, Tanishima T, Yoshimasu N: Relationship between drainage catheter location and postoperative recurrence of chronic subdural hematoma after burr-hole irrigation and closed-system drainage. J Neurosurg 93: 791-795, 2000

15) Boyaci S, Gumustas OG, Korkmaz S, Aksoy K: Endoscopic evacuation of subdural collections. Turk Neurosurg 26: 871-877, 2016

16) Kayaci S, Kanat A, Koksal V, Ozdemir B: Effect of inner membrane tearing in the treatment of adult chronic subdural hematoma: a comparative study. Neurol Med Chir (Tokyo) 54: 363-373, 2014

17) Edlmann E, Giorgi-Coll S, Whitfield PC, Carpenter KLH, Hutchinson PJ: Pathophysiology of chronic subdural haematoma: inflammation, angiogenesis and implications for pharmacotherapy. J Neuroinflammation 14: 108, 2017

18) Inglis K: Subdural haemorrhage, cysts and false membranes; illustrating the influence of intrinsic factors in disease when development of the body is normal. Brain 69: 157-194, 1946

19) Atkinson JL, Lane JI, Aksamit AJ: MRI depiction of chronic intradural (subdural) hematoma in evolution. J Magn Reson Imaging 17: 484-486, 2003

20) Sato S, Suzuki J: Ultrastructural observations of the capsule of chronic subdural hematoma in various clinical stages. J Neurosurg 43: 569-578, 1975

21) Lee KS: History of chronic subdural hematoma. Korean J Neurotrauma 11: 27-34, 2015

22) Holl DC, Volovici V, Dirven CMF, et al.: Pathophysiology and nonsurgical treatment of chronic subdural hematoma: from past to present to future. World Neurosurg 116: 402-411.e2, 2018

23) Gandhoke GS, Kaif M, Choi L, Williamson RW, Nakaji P: Histopathological features of the outer membrane of chronic subdural hematoma and correlation with clinical and radiological features. J Clin Neurosci 20: 1398-1401, 2013

24) Park MH, Kim CH, Cho TG, Park JK, Moon JG, Lee HK: Clinical features according to the histological types of the outer membrane of chronic subdural hematoma. Korean J Neurotrauma 11: 70-74, 2015

25) Niikawa S, Sugimoto S, Hattori T, et al.: Rapid resolution of acute subdural hematoma-report of four cases. Neurol Med Chir (Tokyo) 29: 820-824, 1989

26) Ahn JH, Jun HS, Kim JH, Oh JK, Song JH, Chang IB: Analysis of risk factor for the development of chronic subdural hematoma in patients with traumatic subdural hygroma. J Korean Neurosurg Soc 59: 622-627, 2016

27) Lee KS, Bae WK, Park YT, Yun IG: The pathogenesis and fate of traumatic subdural hygroma. $\mathrm{Br} J$ Neurosurg 8: 551-558, 1994

28) Soleman J, Nocera F, Mariani L: The conservative and pharmacological management of chronic subdural haematoma. Swiss Med Wkly 147: w14398, 2017

29) Satoshi U, Hidehiro O, Chihiro K, Madoka I, Katsutoshi A, Kiyotaka F: Role of saireito in postoperative chronic subdural hematoma recurrence prevention. J Tradit Med 29: 137-142, 2012

30) You W, Zhu Y, Wang Y, et al.: Prevalence of and risk factors for recurrence of chronic subdural hematoma. Acta Neurochir (Wien) 160: 893-899, 2018

31) Han MH, Ryu JI, Kim CH, Kim JM, Cheong JH, Yi HJ: Predictive factors for recurrence and clinical outcomes in patients with chronic subdural hematoma. J Neurosurg 127: 1117-1125, 2017

Address reprint requests to: Yukinari Kakizawa, MD, PhD, Department of Neurosurgery, Suwa Red Cross Hospital, 5-11-50 kogandori, Suwa, Nagano, Japan. e-mail: ykakizawajp@yahoo.co.jp 